\title{
Thiazolidinediones/PPAR $\gamma$ agonists and fatty acid synthase inhibitors as an experimental combination therapy for prostate cancer
}

\author{
MAHMOUD MANSOUR ${ }^{1}$, DEAN SCHWARTZ ${ }^{1}$, ROBERT JUDD ${ }^{1}$, BENSON AKINGBEMI $^{1}$, \\ TIM BRADEN ${ }^{1}$, EDWARD MORRISON ${ }^{1}$, JOHN DENNIS ${ }^{1}$, FRANK BARTOL $^{1}$, \\ AMANDA HAZI $^{1}$, INDIA NAPIER ${ }^{1}$ and ASIM B. ABDEL-MAGEED ${ }^{2}$ \\ ${ }^{1}$ Department of Anatomy, Physiology and Pharmacology, College of Veterinary Medicine, Auburn University, \\ Auburn, AL 36849; ${ }^{2}$ Urology and Oncology Research Laboratories, Tulane University Health \\ Sciences Center, Tulane Cancer Center, New Orleans, LA 70112, USA
}

Received August 18, 2010; Accepted October 8, 2010

DOI: $10.3892 /$ ijo.2010.877

\begin{abstract}
The prostate cancer (PCa) cell lines LNCaP, PC-3, and DU-145 express peroxisome proliferator-activated receptor $\gamma(\mathrm{PPAR} \gamma)$ but its role in PCa is unclear. Thiazolidinediones (TZDs), a family of PPAR $\gamma$ activators and type 2 anti-diabetic drugs, exhibit anti-tumor apoptotic effects in human PCa cell lines. Likewise, pharmacological inhibitors of fatty acid synthase (FASN), a metabolic enzyme highly expressed in $\mathrm{PCa}$, induce apoptosis in prostate and other cancer cells. Here, we show positive correlation between PPAR $\gamma$ and FASN protein in PCa cell lines and synergism between TZDs and FASN blockers in PCa cell viability reduction and apoptosis induction. Combined TZDs/FASN has enhanced anti-tumor properties in both androgen-dependent LNCaP and androgen-independent PC-3 and DU-145 cells when compared with single drug exposure. Low concentrations $(5-10 \mu \mathrm{M})$ of the TZD drug rosiglitazone failed to alter cell viability but, paradoxically, upregulated lipogenic genes [PPAR $\gamma$, FASN, sterol regulatory element binding protein-1c (SREBP-1c) and acetyl-Co A carboxylase-1 (ACC1)], which diminish the apoptotic effects of rosiglitazone. The mean $\mathrm{IC}_{50}$ in all cell lines was $45 \pm 2 \mu \mathrm{M}$ for rosiglitazone compared with significantly lower $5 \pm 1 \mu \mathrm{M}$ for rosiglitazone plus the FASN blocker cerulenin, and 10.2 $\pm 2 \mu \mathrm{M}$ for rosiglitazone plus the cerulenin synthetic analog C75. The $\mathrm{IC}_{50}$ for the combined rosiglitazone and FASN blockers contrasts with the relatively higher $\mathrm{IC}_{50}$ for rosiglitazone $(45 \pm 2 \mu \mathrm{M})$, the TZD drug troglitazone $(13 \pm 2 \mu \mathrm{M})$, cerulenin
\end{abstract}

Correspondence to: Dr Mahmoud Mansour, Department of Anatomy, Physiology and Pharmacology, College of Veterinary Medicine, Auburn University, Auburn, AL 36849, USA E-mail: mansoma@auburn.edu

Key words: thiazolidinediones, prostate cancer, peroxisome proliferator-activated receptor $\gamma$, fatty acid synthase, FASN inhibitors
$(32 \pm 1 \mu \mathrm{M})$, or $\mathrm{C} 75(26 \pm 3 \mu \mathrm{M})$ when these drugs were used alone. In summary, this study shows proof-of-principle for combining FASN blockers and TZDs for PCa treatment.

\section{Introduction}

Prostate cancer (PCa) is second to lung cancer as a cause of cancer-related death in American men (1). Multiple preclinical studies show anti-cancer effects of thiazolidinediones (TZDs) PPAR $\gamma$ agonist ligands (2-6). In contrast, clinical studies suggest that TZDs are largely ineffective as monotherapeutic agents in treating PCa (7). Because cancer cells modify several transduction pathways to achieve continuous progression and survival (8), it is important that multiple drug-strategies are used to achieve effective treatment. Such a strategy should allow for synergistic anti-proliferative effects and/or permit the use of low drug doses that might otherwise be less effective when used as monotherapy (7). For example, the antiproliferative effects of the tyrosine kinase inhibitor gefitinib in A549 lung cancer cells is enhanced with rosiglitazone as the result of rosiglitazone-induced augmentation of PTEN (phosphatase and tensin homolog) expression (9). PTEN inhibits the phosphatidylinositol 3-kinase (PI3-K)-Akt pathway, which is essential for progression of PCa cells. Likewise, the combination of rosiglitazone and the platinum cytotoxic drugs (carboplatin and cisplatin) synergistically inhibit the growth of A549 lung cancer cells compared with single-agent therapy (10). The latter effect appears to be due to down-regulation of metallothioneins that mediate platinum drug resistance.

TZDs induce apoptosis in both androgen-dependent and hormone-refractory tumors and modulate PPAR $\gamma$-target genes that regulate cell cycle, such as cyclin D1 and E (reviewed in ref. 11). Despite TZDs anti-tumor properties, several shortcomings remain that hinder their development as effective treatment for PCa. First, when TZDs are used in vivo as antidiabetic agents they principally regulate genes involved in lipid metabolism and, in turn, increase fat cell numbers and consequently weight gain via genomic pathways (12). Second, their anti-tumor effects require the use of high concentrations 
that could trigger in vivo side effects. Third, troglitazone which is more effective than rosiglitazone in induction of apoptosis is banned in the US due to liver toxicity. Rosiglitazone and pioglitazone which are currently marketed as antidiabetic drugs have failed to suppress PCa cell growth in LNCaP, PC-3 and DU-145 when tested at a single concentration of 10 and $20 \mu \mathrm{M}$, respectively (13). Fourth, in phase II clinical trials rosiglitazone and troglitazone failed to effectively reduce prostate specific antigen (PSA), a tumor marker in prostate cancer (reviewed in ref. 7). Despite these setbacks the TZDs could remain valuable if their toxic or weak monotherapeutic effects are ameliorated through either structural changes or combinational therapy. For example, the current development of TZD derivatives that are similar in structure but devoid of PPAR $\gamma$ activity is one approach that could prove effective in suppression of PCa cells with more potency than the parent drugs (2). Alternatively, in this study we attempted the use of FASN blockers mycotoxin cerulenin and C75 ( $\alpha$-methylene- $\gamma$-butyrolactone) (14-16) to augment the apoptotic effect of rosiglitazone and blunt the induction of fatty acid synthase (FASN), which is induced by genomic effect of rosiglitazone, and by androgens, which are pivotal for growth and differentiation of prostate cells (17).

FASN (EC 2.3.1.85) is an anabolic lipogenic enzyme with seven multifunctional enzymatic sites that regulate de novo fatty acid synthesis (18). It is classified as an oncogene (OA519) because it is highly expressed in prostate (19-22) and other cancers (23). In the presence of NADPH cofactor, the FASN catalyzes the conversion of acetyl-CoA and malonylCoA into long-chain saturated fatty acids such as palmitate (24). Increased FASN activity is thought to drive production of lipids that are essential building blocks for cell membrane and modification of phosphoproteins (18). More importantly, increased expression of FASN protects cancer cells from apoptosis, and is associated with aggressive forms of prostate cancers (25). In support of FASN tumor enhancing properties, silencing of FASN by small interference RNA (siRNA) molecules induces apoptosis in androgen-dependent LNCaP PCa cells (26). Likewise, chemical inhibitors of FASN induce apoptosis in several tumor cell lines $(14,23,27-29)$. In addition to anti-tumor properties of FASN blockers, inhibition of FASN is associated with sustained body weight loss (30). The antitumor and anti-obesity properties of FASN blockers thus provide a rational basis for combinational therapy with TZDs for treatment of both androgen-dependent and hormone refractory $\mathrm{PCa}$. The objectives of this study are 2-fold, i) to investigate the role of FASN and PPAR $\gamma$ in anti-tumor activity of rosiglitazone, and ii) to evaluate the anti-tumor potency of TZDs and FASN inhibitors combinations. We hypothesize that the combined use of TZDs and FASN blockers enhances their antitumor properties in $\mathrm{PCa}$.

\section{Materials and methods}

Reagents. Rosiglitazone, troglitazone, GW 9662 (PPAR $\gamma$ blocker) were purchased from Cayman Chemical (Ann Arbor, MI). The antibiotic Cerulenin, C75 ( $\alpha$-methylene- $\gamma$-butyrolactone), mouse antibody against $\beta$-actin, DMSO, 5 - $\alpha$-dihydrotestosterone ( $5 \alpha-\mathrm{DHT})$, and trypsin were from Sigma (St. Louis, MO). For culture experiments all stock drugs were prepared at various concentrations in DMSO, further diluted in phenol red-free RPMI-1640 media and added in culture to give $0.1 \%$ final DMSO concentration. Mouse monoclonal antiPPAR $\gamma$ (sc-7273, E8), FASN polyclonal antibody (sc-20140, $\mathrm{H}-300$ ), and secondary mouse and rabbit antibodies were obtained from Santa Cruz Biotechnology, Inc. (Santa Cruz, CA). RPMI- 1640 media with and without phenol red and penicillin-streptomycin antibiotics were purchased from Invitrogen Life Technologies Invitrogen Inc. (Carlsbad, CA). Heat-inactivated and charcoal-dextran-treated fetal calf serum (FCS) was obtained from Atlanta Biological (Atlanta, GA). Cell culture flasks and other supplies were purchased from VWR International, LCC (Atlanta, GA).

Cell culture. The human androgen-dependent (LNCaP) and androgen-independent (PC-3 and DU-145) PCa cells were obtained from the American Type Culture Collection (Manassas, VA). Cells were maintained in RPMI-1640 media supplemented with $1 \%(\mathrm{v} / \mathrm{v})$ streptomycin-penicillin antibiotics (Invitrogen), and 10\% (v/v) heat-inactivated FCS. Cells were grown in T-75 vented filter cap tissue culture flasks until they reach approximately $75-90 \%$ confluency at $37^{\circ} \mathrm{C}$ in a humidified incubator under $5 \%$ carbon dioxide atmosphere. Twenty-four hours prior to drug treatment, the culture media was aspirated and replaced with phenol red-free RPMI-1640 supplemented with $5 \%$ charcoal-dextran-treated FCS. Cells were seeded at a density of $2 \times 10^{6}$ cells per well when grown in 6-well culture plates, or $1 \times 10^{6}$ cells per wells in 24-well plates, and at $0.1 \times 10^{5}$ cells per well when grown in flat-bottom 96-well plates. Cells were used between passages 5 and 38 .

MTT cell viability assay. Cells in log-phase growth were harvested via trypsinization and counted using a coulter counter. Effect of test drugs on cell viability was determined using the 3-(4,5-dimethylthiazol-2-yl)-2-5-diphnyltetrazolium bromide (MTT) cell viability assay [American Type Culture Collection (ATCC)] according to the manufacturer's instructions. The assay measures the reduction of a tetrazolium component (MTT) into an insoluble formazan product by mitochondria of viable cells. Forty-eight hours after test drugs were added (four replicates per each drug concentration using 96-well plates), $10 \mu 1$ MTT reagent was added per well for $2 \mathrm{~h}$ at $37^{\circ} \mathrm{C}$. Next, $100 \mu 1$ detergent solution was added to lyse the cells and solubilize colored crystals. Plates were then incubated in the dark for $6 \mathrm{~h}$ at room temperature. Optical density values for samples were obtained using Dynex Technologies MRX-TC revelation microtiter plate reader at $570 \mathrm{~nm}$ wavelength. Media plus DMSO (vehicle) was used as blank. The amount of color produced is directly proportional to the number of viable cells. Concentrations that result in $50 \%$ inhibition $\left(\mathrm{IC}_{50}\right)$ of cell viability were calculated using SigmaStat and Sigma Plot software (San Diego, CA). Calculations of mean $\mathrm{IC}_{50}$ were based on data from three independent experiments.

Determination of apoptosis by ELISA. Apoptosis induced by drug treatments was determined with a Cell Death Detection ELISA kit (Roche Diagnostics) assay according to the manufacturer's instruction. The ELISA test assay is based on the quantitative determination of cytoplasmic histone-associated 
DNA fragments in the form of mononucleosomes and oligonucleosomes following apoptotic death induction. Briefly, $0.1 \times 10^{5}$ cells were cultured in 24 -well plates in complete RPMI-1640 with $10 \%$ fetal bovine serum for $24 \mathrm{~h}$. The next day, the media were aspirated and replaced with phenol redfree RMPI-1640 supplemented with charcoal-treated FCS. Cells were subsequently treated with test drugs at various concentrations for $24 \mathrm{~h}$. For apoptosis determination both adherent and floating cells were tested immediately after collection. Cell lysates equivalent to approximately $0.5 \times 10^{5}$ cells were used in the ELISA.

Conventional and real-time PCR. Total RNA was isolated using TRIzol reagent (Invitrogen-Life Technologies Inc., Carlsbad, CA, USA), according to the manufacturer's protocol and as described previously in our laboratory (31). RNA concentrations were determined at $260 \mathrm{~nm}$ wavelength and the ratio of $260 / 280$ was obtained using UV spectrophotometry (DU640, Beckman Coulter Fullerton, CA, USA). Samples with $260 / 280$ ratio of $\geq 1.8$ were used. First strand synthesis was accomplished with SABiosciences first strand kit C-03 (SABiosciences, Frederic, MD, USA).

Conventional PCR was used initially to determine the presence of lipogenic genes in LNCaP, PC-3, and DU-145 using validated primer sets synthesized by Integrated DNA Technology (San Diego, CA). RT-PCR was performed using Reaction Ready ${ }^{\mathrm{TM}}$ Hot Start 'Sweet' PCR Master Mix (SABiosciences). PCR products were analyzed in parallel with ribosomal 18s (Ribo s18) housekeeping gene. Final end PCR products were fractionated and visualized on $2 \%$ ethedium bromide-stained agarose gel. Next, real-time PCR was used to determine the expression level of the lipogenic genes in LNCaP cells treated with low $(5 \mu \mathrm{M})$ and high rosiglitazone concentration $(50 \mu \mathrm{M})$. Real-time PCR was performed as we previously described (31). Briefly, reactions were performed in $25 \mu \mathrm{l}$ reaction mixture containing $12.5 \mu \mathrm{l} \mathrm{RT} 2$ real-time SYBR/Fluorescein Green PCR master mix with final concentrations of $10 \mathrm{mM}$ Tris- $\mathrm{HCl}, 50 \mathrm{mM} \mathrm{KCl}, 2.0 \mathrm{mM} \mathrm{MgCl}_{2}$, $0.2 \mathrm{mM}$ dNTPs, and 2.5 units of HotStart Taq DNA polymerase (SABiosciences), $1 \mu 1$ first strand cDNA, $1 \mu 1 \mathrm{RT} 2$ validated primer sets, and $10.5 \mu \mathrm{l} \mathrm{PCR}$-grade water. Reactions were run in 96-well PCR plates using Bio-Rad PCR cycler (Bio-Rad, MyiQ, Hercules, CA, USA). All reactions were run in duplicates and the results were normalized to Ribo 18s housekeeping-gene. The amplification protocol was set at $95^{\circ} \mathrm{C}$ for $15 \mathrm{~min}$, and 40 cycles each at $95^{\circ} \mathrm{C}$ for $30 \mathrm{sec}, 55^{\circ} \mathrm{C}$ for $30 \mathrm{sec}$, and $72^{\circ} \mathrm{C}$ for $30 \mathrm{sec}$.

Western blot analysis. Western blot analysis was used to determine protein expression for PPAR $\gamma$ and FASN in LNCaP, $\mathrm{PC}-3$, and DU-145. Cells grown in 6-well plates were washed in $\mathrm{Ca} / \mathrm{Mg}$ free PBS and then lysed by scraping in 100-250 $\mu \mathrm{l}$ T-PER Tissue Protein Extraction Reagent (Pierce, Rockford, IL) containing EDTA, protease and phosphatase inhibitor cocktail. Total protein concentration was determined in $2 \mu \mathrm{l}$ aliquot using Bradford Bio-Rad microplate assay protocol. An equal volume of protein sample $(30 \mu \mathrm{g})$ was mixed with an equal volume of $2 \mathrm{X}$ SDS-PAGE sample loading buffer (100 mmol/1 Tris-HCl pH 6.8, 4\% SDS, 5\% ß-mercaptoethanol, $20 \%$ glycerol, and $0.1 \%$ bromophenol blue). Samples were boiled for $5 \mathrm{~min}$ and equal amounts of protein were loaded per well in $10-12 \%$ precast SDS-PAGE gels (Bio-Rad). Next, protein samples were transferred to nitrocellulose membranes for over $90 \mathrm{~min}$, using Bio-Rad mini trans-blot electrophoretic transfer cell. Following electrical transfer of proteins, membranes were blocked with TBS containing $0.05 \%$ Tween 20 (TBS-T) and 5\% non-fat dry milk. Target proteins were detected using appropriate primary antibodies diluted in blocking buffer (1:300 for FASN and 1:400 for PPAR $\gamma$ ). Blots with primary antibodies were incubated overnight at $4{ }^{\circ} \mathrm{C}$ with continuous shaking. The next day, the membranes were washed $3 \mathrm{x}$ for $15 \mathrm{~min}$ each and incubated with HRP-labeled secondary antibodies (1:5000 for HRP-conjugated goat anti-rabbit, and 1:2000 for goat anti-mouse antibodies) for $90 \mathrm{~min}$. Next, membranes were washed $3 \mathrm{x}$ for $10 \mathrm{~min}$ with $0.05 \%$ Tween 20 in TBS. Detection of specific binding was determined with ECL substrate (1-3 min). Excess detection mixture was then removed and the blots, which were wrapped in protective sheets, were exposed to X-ray film. For correlation between PPAR $\gamma$ and FASN protein in cell lines, the band intensity for each protein was determined from replica generated from three experiments using the Un-Scan-It software (Silk Scientific, Inc., Orem, UT, USA).

Statistical analysis. Data from cell culture MTT cell viability assay were expressed as mean \pm SEM. The data were analyzed by Student's t-test, one-way ANOVA, followed by Tukey post hoc test (Graph Pad Prism 5.0, San Diego, CA). A P $<0.05$ was considered statistically significant. Statistical analysis of real-time PCR data was performed using a modification of the $\Delta \Delta \mathrm{Ct}$ method $(\Delta \Delta \mathrm{Ct})$ as described previously (32).

\section{Results}

PPARy expression in PCa cell lines parallels that of FASN. As an initial step for use of TZDs PPAR $\gamma$ agonists and FASN inhibitors as anti-tumor drugs, we compared FASN expression with that of PPAR $\gamma$ in PC-3, DU-145, and LNCaP cell lines. Because PPAR $\gamma$ is expressed in a cell line-dependent manner (higher in androgen-independent than androgen-dependent cells) and its activation via rosiglitazone induces FASN expression in vivo (33), we hypothesize that the expression of FASN would parallel PPAR $\gamma$ levels in untreated PC-3, DU-145 and LNCaP cells. As shown in Fig. 1A-C, FASN protein was expressed at a higher level in PC-3 and DU-145 compared with expression levels in $\mathrm{LNCaP}$ and normal prostate (NP) tissue. Likewise, PPAR $\gamma$ levels were higher in P-C3 and DU-145 androgen-independent $\mathrm{PCa}$ cells compared with expression in androgen-dependent LNCaP cells and NP, Fig. 1C. More importantly, the intensity of FASN protein expression positively correlated with that of PPAR $\gamma$ in a tissue-dependent manner. The correlation coefficient $(\mathrm{R})$ calculated from blotting the expression level of PPAR $\gamma$ protein against that of the FASN was 0.98 with $p=0.0123$. These data suggest that the two proteins tend to increase or decrease together. In the next set of experiments, we investigated the contribution of PPAR $\gamma$ to rosiglitazone and troglitazone anti-tumor activity.

Anti-tumor activity of rosiglitazone and troglitazone in $P C a$ cells is independent of PPARy except at low rosiglitazone 
A

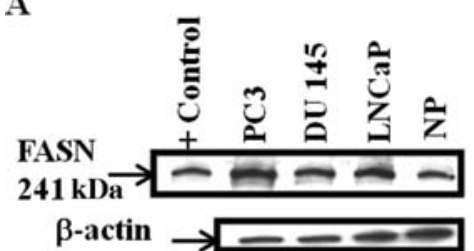

B

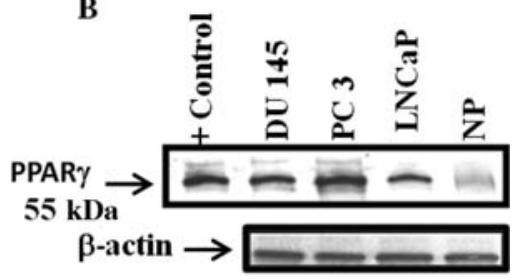

C

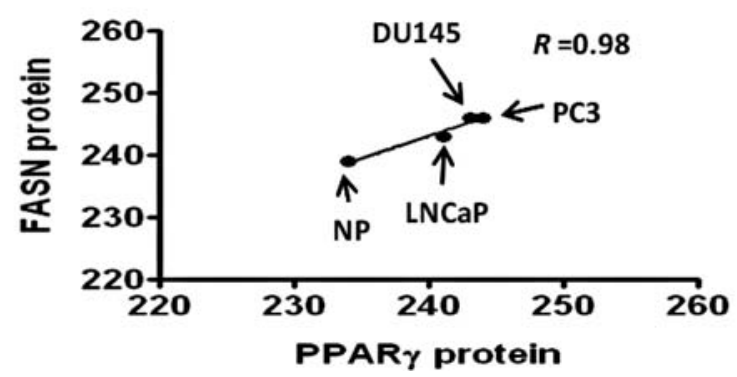

Figure 1. Fatty acid synthase (FASN) protein strongly correlated with peroxisome proliferator-activated receptor $\gamma(\mathrm{PPAR} \gamma)$ protein expression in prostate cancer (PCa) cell lines. Figure shows representative Western blots for FASN (241 kDa) (A) and PPAR $\gamma$ (55 kDa) (B) proteins in PC-3, DU-145, LNCaP cell lines, and normal prostate (NP) tissue. Correlation between FASN and PPAR $\gamma$ proteins was determined by measurement of band densitometry generated from three independent experiments as shown in (C). The correlation coefficient (R) between PPAR $\gamma$ and FASN proteins was 0.98 with p=0.0123 (C). FASN and PPAR $\gamma$ expression were higher in androgen-independent PC-3 and DU-145 compared with androgen-dependent LNCaP and NP. A549 carcinoma human alveolar basal epithelial cell lysate was used as positive control for FASN protein, and 3T3-L1 cell lysate for PPAR $\gamma$ protein.
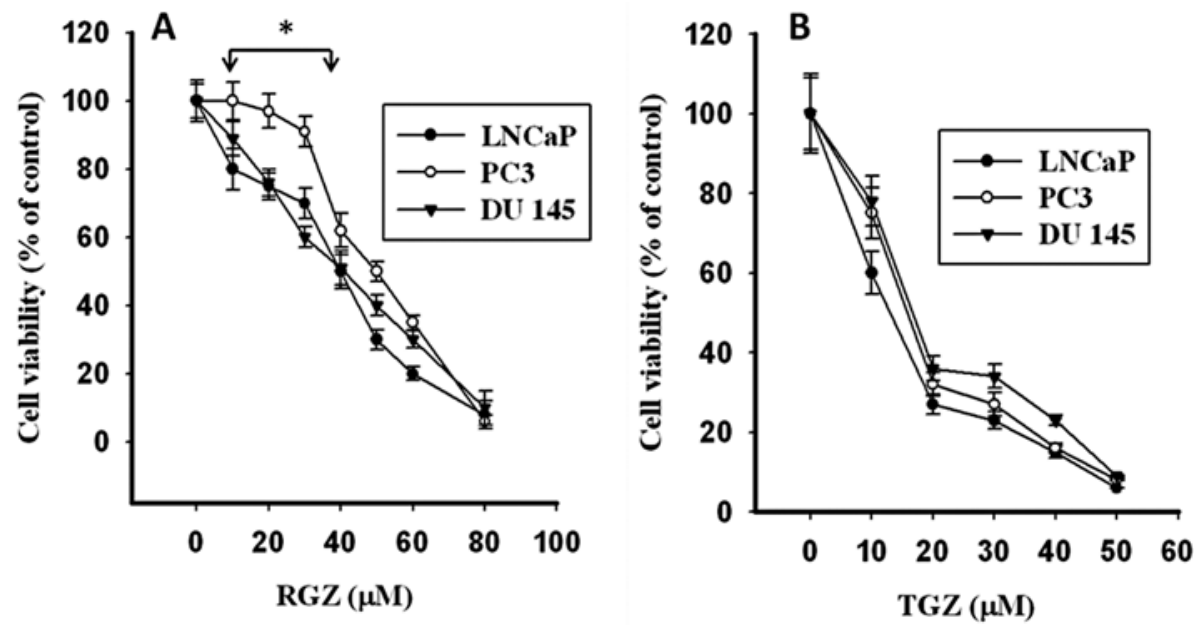

Figure 2. Androgen-independent PC-3 cells that have the highest expression of FASN and PPAR $\gamma$ proteins were significantly less sensitive to anti-tumor effects of rosiglitazone (RGZ) concentrations below $30 \mu \mathrm{M}$. Figure shows \% cell viability of LNCaP, DU-145, and PC-3 cells treated with RGZ (A) or TGZ (B) using multiple drug concentrations. Data are shown as mean \pm SEM. Asterisk denotes statistical significance of PC3 compared to LNCaP (P<0.05).

concentration. Rosiglitazone has potent PPAR $\gamma$ activation effects compared to troglitazone. Due to higher expression of FASN and PPAR $\gamma$ in androgen-independent PCa cells, especially PC-3, versus androgen-dependent LNCaP cells (Fig. 1), the objective of this set of experiments was to determine if the anti-tumor activity of rosiglitazone and troglitazone will be affected by variable PPAR $\gamma$ levels in the aforementioned three cell lines. This experiment was conducted under androgen-depleted conditions. As shown in Fig. 2A, a weaker anti-tumor activity was observed for rosiglitazone in PC-3 cells at the lower range of rosiglitazone concentrations (10-30 $\mu \mathrm{M})$. At higher concentrations (40 $\mu \mathrm{M}$ and above), there was no difference in the anti-tumor activity of rosiglitazone between the cell lines. In contrast to rosiglitazone, the anti-tumor activity of troglitazone was not altered by constitutively higher expression levels of FASN and PPAR $\gamma$ in PC-3 cells (Fig. 2B). Compared with rosiglitazone, troglitazone consistently inhibited cell viability in a concentration-dependent manner, irrespective of the cell line used. The average $\mathrm{IC}_{50}$ calculated from three independent experiments using data from all three cell lines was $45 \pm 2 \mu \mathrm{M}$ for rosiglitazone, and $13 \pm 2 \mu \mathrm{M}$ for troglitazone. The weaker anti-tumor activity of rosiglitazone at the lower concentration range (especially in PC3) versus at higher concentrations suggests two mechanisms 


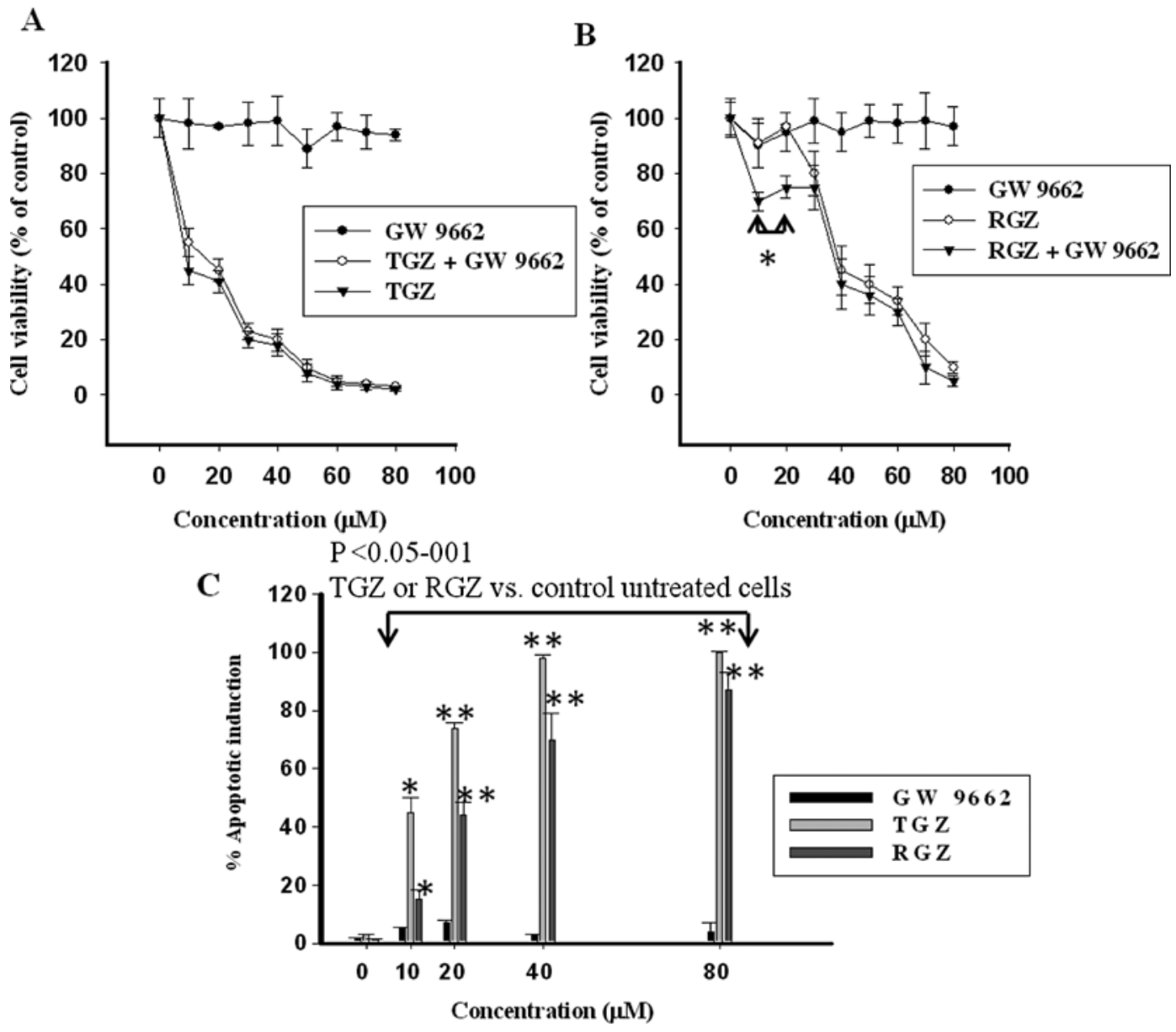

Figure 3. Blockade of PPAR $\gamma$ in PC3 cells by GW 9662 irreversible PPAR $\gamma$ blocker does not alter cell viability (A or B) or reverse troglitazone (TGZ) suppressive effects on cell viability (A). Pretreatment of PC3 with PPAR $\gamma$ GW 9962 blocker enhanced rosiglitazone (RGZ) antitumor activity at lower (10-20 $\mu \mathrm{M}$ ) but not higher concentrations ( $30 \mu \mathrm{M}$ and above) compared with PC3 cells treated with RGZ alone (B). The \% induction of apoptosis by GW 9962, TGZ and RGZ at selected concentrations mimicked their suppressive effects on cell viability (C). Cells in (A and B) were treated with GW 9962 for 30 min before TGZ or RGZ was added. Data are shown as mean \pm SEM. Asterisks denote statistical significance $\left({ }^{*} \mathrm{p}<0.05,{ }^{* *} \mathrm{p}<0.001\right)$.

of actions of rosiglitazone that might involve genomic and nongenomic effects at lower and higher concentrations, respectively.

GW 9662 PPAR blocker suggests two mechanisms of action for rosiglitazone in PCa but not for troglitazone. The role of PPAR $\gamma$ in PCa is unknown and in certain instances PPAR $\gamma$ is suggested to stimulate cancer formation (11). As rosiglitazone is a potent activator of PPAR $\gamma$ and FASN, this prompted us to determine if blockade of PPAR $\gamma$ in PC-3 cells modulated rosiglitazone anti-tumor actions. In the following experiments GW 9662, an irreversible PPAR $\gamma$ blocker, and TZDs were evaluated for their effects on cell growth and induction of apoptosis. Blockage of PPAR $\gamma$ per se using various GW 9662 concentrations did not alter cell viability (Fig. 3A and B). Likewise, blockade of PPAR $\gamma$ has failed to reverse the antitumor effects of troglitazone in PC-3 cells (Fig. 3A). On the other hand, PPAR $\gamma$ blockade in PC-3 enhanced the anti-tumor activity of rosiglitazone at lower rosiglitazone concentration range (10-20 $\mu \mathrm{M})$ (Fig. 3B). This effect of GW 9662 is blunted at higher micromolar range ( $30 \mu \mathrm{M}$ and above) and is not apparent with troglitazone, suggesting genomic and non-genomic actions of rosiglitazone at lower and higher concentrations, respectively. To further investigate the anti- tumor activity of rosiglitazone at lower and higher concentrations, we determined the ability of TZDs rosiglitazone and troglitazone, and GW 9662 to induce apoptosis in PC-3 cells. As shown in Fig. 3C, the apoptotic properties of these compounds parallel their suppressive effects on cell viability shown in Fig. 3A and B. In summary, data from these experiments suggest that PPAR $\gamma$ activation contributes to lower anti-tumor activity of rosiglitazone when used at lower micromolar concentrations compared with troglitazone. As the blockade of PPAR $\gamma$ enhances rosiglitazone anti-tumor action at lower concentrations (but not at higher concentrations) this finding adds further evidence for the hypothesis of two mechanisms of actions for rosiglitazone.

Induction of FASN by low rosiglitazone concentration (genomic effect) may contribute to apoptotic ineffectiveness of rosiglitazone when used as a single drug agent. To further investigate the mechanism of weaker anti-tumor and antiapoptotic activity of rosiglitazone at low micromolar concentration (Fig. 3), we hypothesize that rosiglitazone induces the anti-apoptotic enzyme FASN by activation of PPAR $\gamma$. This may partly explain the weaker anti-apoptotic effect of rosiglitazone. Several observations support the concept of 

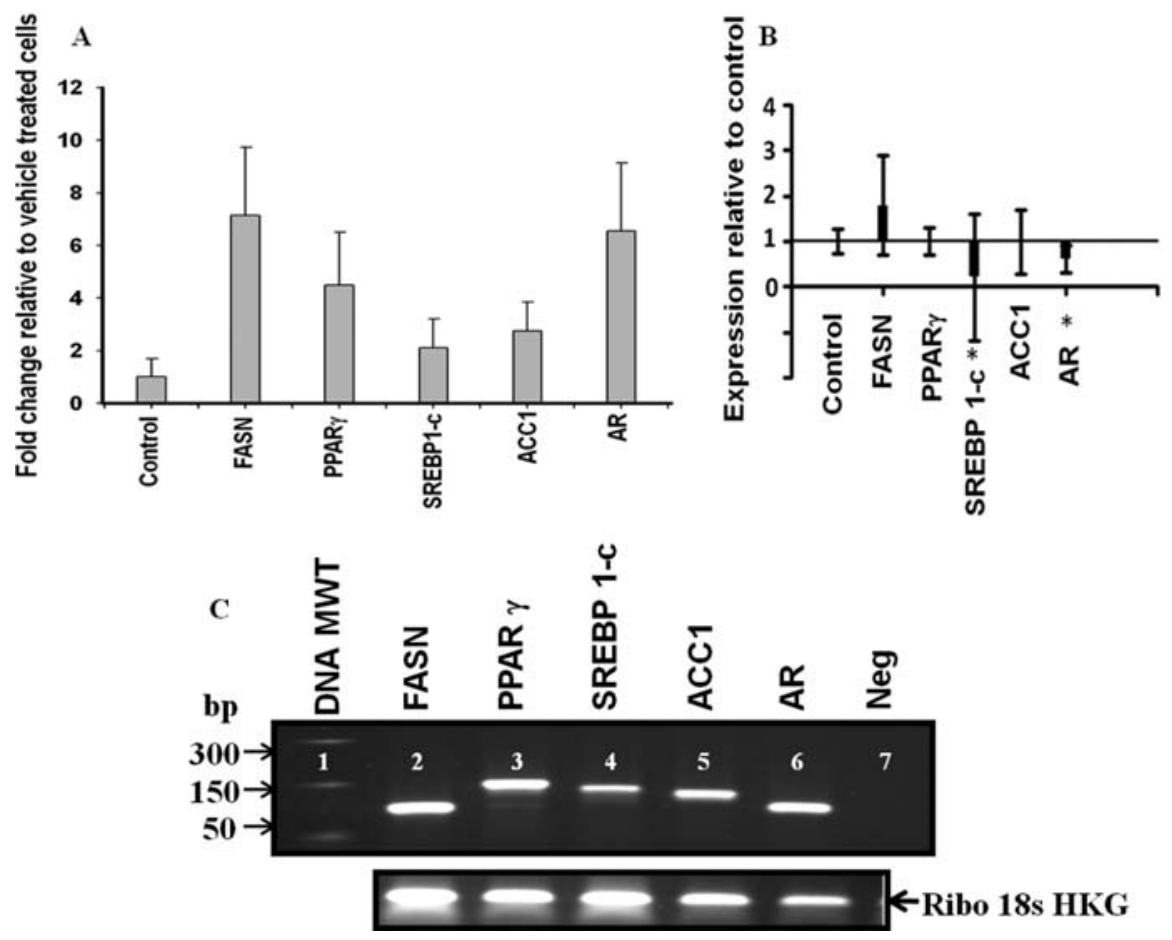

Figure 4. Rosiglitazone (RGZ) exhibits a biphasic effect on induction of lipogenic genes in androgen-dependent LNCaP cells. At $5 \mu \mathrm{M}$ and under androgendeprived conditions, RGZ upregulated lipogenic gene expression (A) but this effect was abolished at higher $(50 \mu \mathrm{M})$ concentration (B). Exposure (5 $\mu \mathrm{M})$ increased expression of FASN, PPAR $\gamma$, SREBP-1c, ACC1, and AR mRNA by 7, 4.2, 2, 2.3, and 6-fold, respectively (A). In contrast, the expression was unchanged (for FASN, PPAR $\gamma$ and ACC1) or significantly decreased (for SREBP 1-c and AR) at $50 \mu \mathrm{M} \mathrm{RGZ} \mathrm{treatment} \mathrm{(B).} \mathrm{Genes} \mathrm{were} \mathrm{normalized} \mathrm{with}$ Ribo18s housekeeping gene and expressed relative to control untreated cells (set at 1). The specificity of the primers was confirmed by verification of the expected product size using conventional RT-PCR (C). Real-time PCR data (A and B) are shown as mean \pm SD. Expected PCR product size is shown in Table I. Asterisk (B) denotes statistical significance ( $\left.{ }^{*} \mathrm{p}<0.05\right)$.

Table I. Expected product size and accession numbers of genes used in the study.

\begin{tabular}{lcl}
\hline Gene & Expected product size & Accession no. \\
\hline FASN & 94 & NM_004104 \\
PPAR $\gamma$ & 181 & NM_015869 \\
SREBP-1c & 131 & NM_004176 \\
ACC1 & 124 & NM_198834 \\
AR & 98 & NM_000044 \\
\hline
\end{tabular}

Genes assayed include fatty acid synthase (FASN), peroxisome proliferator-activated receptor $\gamma(\operatorname{PPAR} \gamma)$, sterol regulatory element binding protein-1c (SREBP-1c), acetyl-Co A carboxylase-1 (ACC1), and androgen receptor (AR).

PPAR $\gamma$-induced induction of FASN, and the anti-apoptitic properties of the latter enzyme. First, overexpression of the lipogenic gene FASN enzyme has been shown to protect $\mathrm{PCa}$ cells against induction of apoptosis (25), and under androgen receptor-dependent conditions FASN induced invasive adenocarcinomas in immunodeficient mice (25). Second, PPAR $\gamma$ activation by feeding rosiglitazone in mice increased in vivo FASN production by $>6$-fold (33). Third, we showed that FASN expression levels parallel those of PPAR $\gamma$ at basal levels (Fig. 1). For testing the production of FASN by rosiglitazone treatment we stimulated LNCaP (with the lowest
FASN and PPAR $\gamma$ levels) with low $(5 \mu \mathrm{M})$ and high $(50 \mu \mathrm{M})$ rosiglitazone concentrations and then determined expression of a panel of genes involved in lipogenesis and transcriptional regulation of FASN by real-time PCR. As shown in Fig. 4A, rosiglitazone $(5 \mu \mathrm{M})$ treatment increased the transcriptional activity of PPAR $\gamma$ and lipogenic gene production including that of FASN. In comparison with DMSO stimulated cells (control expression level set at 1), rosiglitazone treatment increased the activity of FASN, PPAR $\gamma$, SREBP-1c, ACC1, and AR by 7, 4.2, 2, 2.3, and 6-fold, respectively. In contrast, at $50 \mu \mathrm{M}$ rosiglitazone concentration, FASN, PPAR $\gamma$ and ACC1 expression was unchanged while that of SREBP 1-c and AR was significantly decreased, Fig. 4B. These data add further support to genomic and non-genomic effects of rosiglitazone when used at low and high concentrations, respectively.

Combined rosiglitazone and FASN blockers cerulenin or its analog C75 have additive suppressive effects on PCa cell growth and apoptosis. FASN pharmacological inhibitors and TZDs have anti-tumor properties when used as single drugs $(14,34,35)$. Because troglitazone is banned from the US market due to idiosyncratic hepatotoxicity (36) and rosiglitazone treatment is associated with increased in vivo adipogenesis (33), and induction of in vitro lipogenic genes, we used rosiglitazone to test the hypothesis that blockade of FASN would enhance the rosiglitazone anti-tumor effect. As shown in Fig. 5A, the combined use of rosiglitazone and the FASN blocker cerulenin or C75 consistently induced significantly higher cell death effects in $\mathrm{LNCaP}$ compared with rosiglitazone 

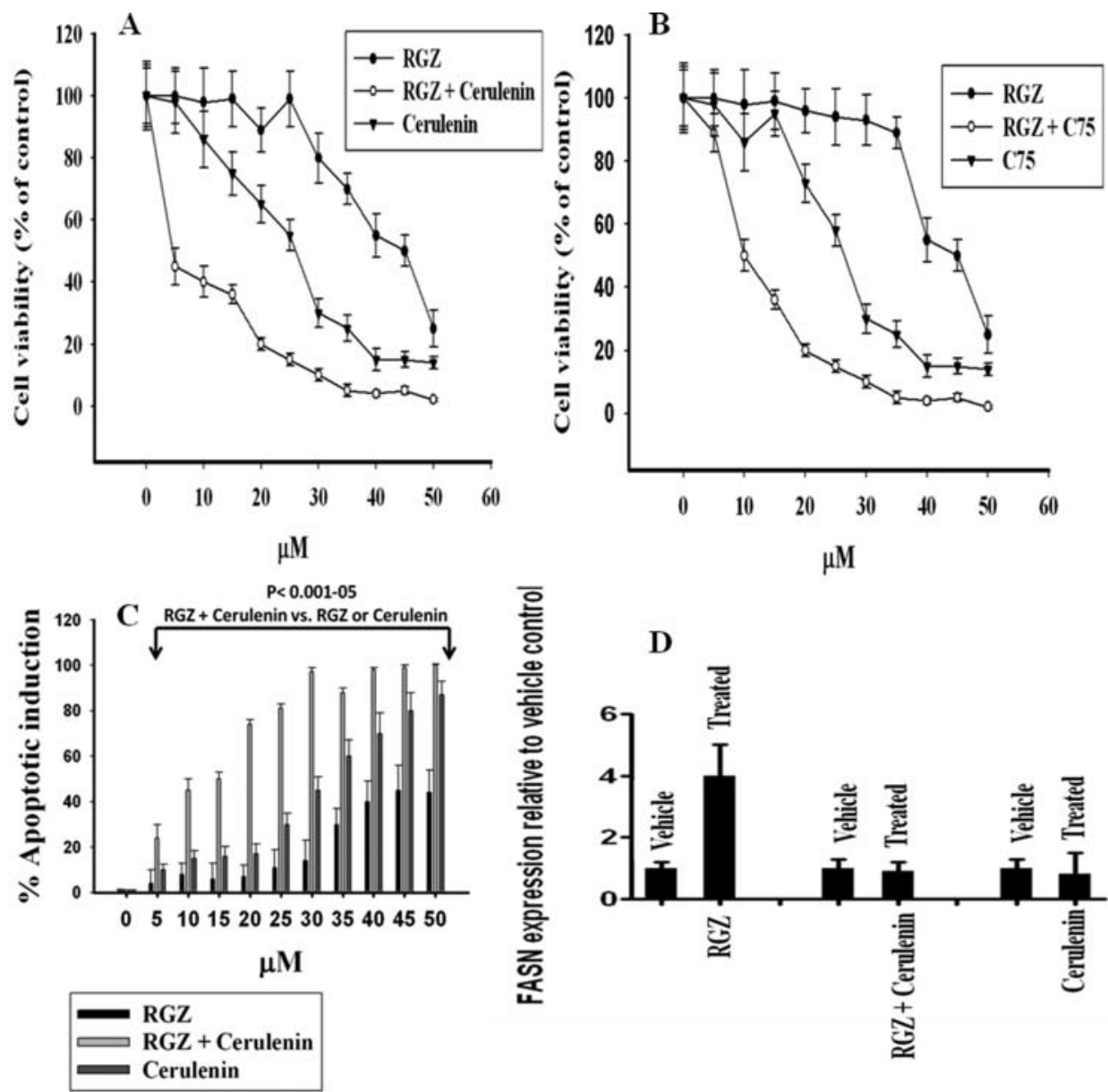

Figure 5. Rosiglitazone (RGZ) combined with irreversible fatty acid synthase (FASN) inhibitor cerulenin (A) or C75 (B) suppresses LNCaP cell viability more potently than each drug alone. The $\mathrm{IC}_{50}$ was $45 \pm 2 \mu \mathrm{M}$ for RGZ, $32 \pm 1 \mu \mathrm{M}$ for cerulenin and $26 \pm 3 \mu \mathrm{M}$ for C75 alone compared with significantly lower $5 \pm 1 \mu \mathrm{M}$ RGZ/cerulenin and $10.2 \pm 2 \mu \mathrm{M}$ RGZ/C75 combinations. The inhibition of cell viability by RGZ/cerulenin combination was verified by measurement of $\%$ apoptosis induction (C) and by transcriptional repression of FASN mRNA by RGZ/cerulenin compared with rosiglitazone alone (5 $\mu$ M) (D). Data are shown as mean \pm SEM. P-values are for the antitumor effect of combined RGZ and FASN blockers versus RGZ vary from 0.001 to 0.05 with the highest difference at lower concentrations.

or FASN blockade alone. The added synergism of FASN irreversible blockers to the anti-proliferative properties of rosiglitazone was seen in a concentration-dependent manner with greater effect occurring at lower rosiglitazone concentrations (Fig. 5A and B). The $\mathrm{IC}_{50}$ for rosiglitazone/cerulenin drug combination was $5 \pm 2 \mu \mathrm{M}$ compared to $45 \pm 2 \mu \mathrm{M}$ for rosiglitazone and $32 \pm 1 \mu \mathrm{M}$ for cerulenin alone. Likewise, the $\mathrm{IC}_{50}$ for rosiglitazone/C75 combination was $10.2 \pm 2 \mu \mathrm{M}$ compared to $45 \pm 2 \mu \mathrm{M}$ and $26 \pm 3 \mu \mathrm{M}$ for rosiglitazone and C75, respectively. Similarly, the induction of apoptosis by rosiglitazone/cerulenin combination, shown in Fig. 5C, is in agreement with the results of cell viability in Fig. 5A where the apoptotic effect of rosiglitazone/cerulenin was greater relative to that induced by rosiglitazone and cerulenin alone. Taken together, the cell viability and apoptosis data suggest that FASN blockade potentiates rosiglitazone anti-tumor effects. More importantly this effect is more evident at lower rosiglitazone concentrations. To further confirm the abolishment of FASN activity, we treated LNCaP cells with rosiglitazone/cerulenin combination $(5 \mu \mathrm{M}$ each) or with cerulenin $(5 \mu \mathrm{M})$ or rosiglitazone $(5 \mu \mathrm{M})$ alone and then determined FASN mRNA production levels with real-time
PCR. In cancer cells, FASN pharmacological blockers induce inhibition of FASN expression (37) and as predicted here, treatment with the rosiglitazone/cerulenin combination or cerulenin alone abolished FASN mRNA production which was seen only with rosiglitazone treatment (Fig. 5D). Overall, the data from these experiments suggest that rosiglitazone/FASN blockers result in enhancement of rosiglitazone anti-tumor activity in PCa.

To test the anti-proliferative synergistic effects of combined rosiglitazone/FASN treatment in androgen-independent PCa cell lines we treated PC-3 and DU-145 with various concentrations of rosiglitazone, $\mathrm{C} 75$, or rosiglitazone plus C75 and then determine cell viability with the MTT assay. Similar to that in LNCaP (Fig. 5B), rosiglitazone/C75 suppressed cell viability in androgen-independent PC-3 and DU-145 more effectively than rosiglitazone or C75 alone (Fig. 6). This is especially evident at the lower concentrations (range 5-20 $\mu \mathrm{M}$ ).

Combined TZDs and FASN blockers inhibit cell viability in androgen-dependent $\mathrm{LNCaP}$ in the presence of dihydrotestosterone $(D H T)$. Androgen is critical in PCa progression and 


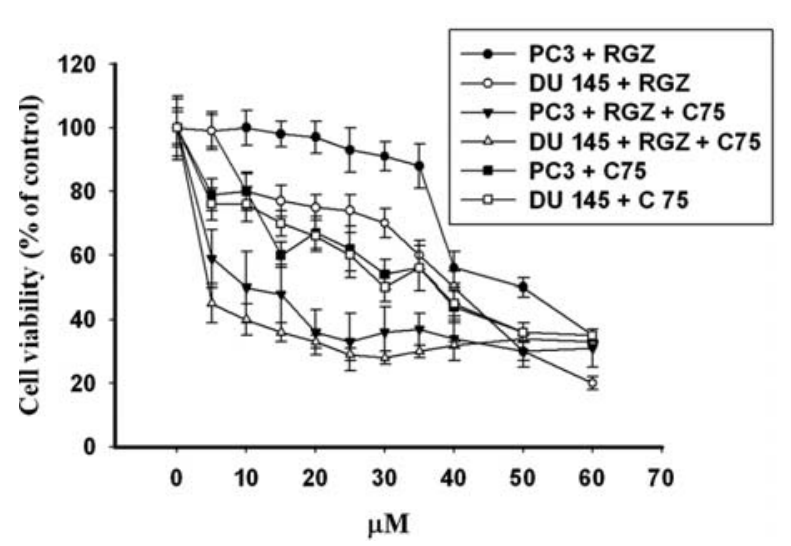

Figure 6. Synthetic fatty synthase inhibitor C75 and the thiazolidinedione drug rosiglitazone (RGZ) are synergistic in repression of cell viability in androgen-independent PC-3 and DU-145 prostate cancer cell lines. Figure shows significantly lowered cell viability in PC3 or DU 145 cells treated with RGZ plus C75 compared with cells treated with RGZ or C75 alone. P-values vary from 0.001 to 0.05 depending on the concentration point.

differentiation of both androgen-dependent and -independent cells. Androgen action in $\mathrm{PCa}$ is accompanied by strong induction of lipogenic genes (38-40). Hence we tested the combined anti-tumor effects of TZDs and FASN blockers in the presence or absence of the non-aromatizable potent androgen 5- $\alpha$-dihydrotestosterone (DHT). As shown in Fig. 7, treatment with TZDs/FASN C75 or TZDs/FASN cerulenin resulted in enhanced suppression of androgen-dependent LNCaP cell viability relative to single drug treatment. Note that rosiglitazone alone, but not troglitazone, failed to alter cell viability when compared with vehicle treated cells. In summary, these data show that the FASN inhibitors cerulenin and C75 enhanced the anti-tumor activity of TZDs under androgen-dependent conditions.

\section{Discussion}

Both TZDs (6) and FASN inhibitors (14) exhibit apoptosismediated anti-tumor effects but the in vivo potency of TZD monotherapy in suppression of PCa cell growth in human clinical trials is weak (reviewed in ref. 7). Although troglitazone exhibits more effective anti-apoptotic effects than rosiglitazone, its liver toxicity has precluded its therapeutic use in humans. In contrast, rosiglitazone has more potent PPAR $\gamma$ activity but weaker apoptotic effects. In this study, we show that the in vitro anti-tumor activity of rosiglitazone is vastly enhanced by novel treatment regimens that combine FASN inhibitors with rosiglitazone. FASN inhibitors (cerulenin or its synthetic analog C75) combined with low rosiglitazone concentrations resulted in enhanced anti-tumor activity both in androgen-independent (PC-3 and DU-145) and androgendependent ( $\mathrm{LNCaP}$ ) $\mathrm{PCa}$ cell lines when tested under androgen-deprived conditions. Likewise, in the presence of the potent androgen DHT, the apoptotic effects of rosiglitazone plus the FASN inhibitors cerulenin or C75 in LNCaP was superior to rosiglitazone, cerulenin or C75. Further, the TZDs plus FASN inhibitors allowed for reciprocal synergistic anti-proliferative effects. The rosiglitazone/FASN drug combinations were formulated with low rosiglitazone and FASN inhibitor concentrations that were ineffective in $\mathrm{PCa}$

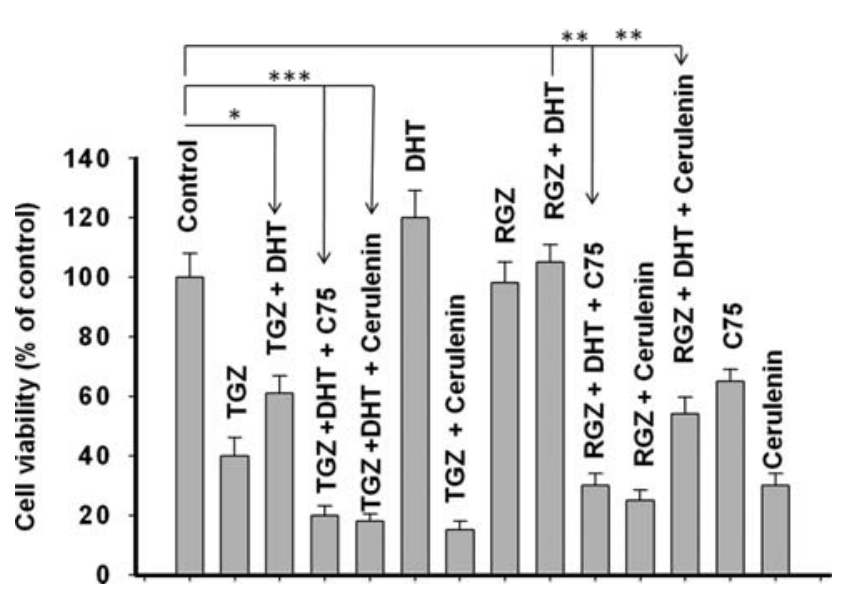

Figure 7. Combining fatty acid synthase (FASN) inhibitors (cerulenin or C75) with the thiazolidinediones (TZDs), rosiglitazone (RGZ) or troglitazone (TGZ), induced greater cell viability inhibition in LNCaP cells in the presence of DHT $(0.1 \mathrm{nM})$ compared with RGZ, TGZ, or FASN inhibitors alone. Data are shown as mean \pm SEM. Levels of significance $\left({ }^{*} \mathrm{p}<0.05\right.$, $\left.{ }^{* *} \mathrm{p}<0.01,{ }^{* * *} \mathrm{p}<0.001\right)$ is shown for TZDs or TZDs plus FASN inhibitors. RGZ and TGZ were added at $13 \mu \mathrm{M}$ each while Cerulenin and C75 were added at $10 \mu \mathrm{M}$ each.

cell suppression when used separately. For example, a 50\% reduction in LNCaP cell viability was obtained with rosiglitazone/cerulenin combination containing $5 \mu \mathrm{M}$ each, while cell viability was unaltered with rosiglitazone $(5 \mu \mathrm{M})$ or cerulenin $(5 \mu \mathrm{M})$. Similar findings were also obtained with the synthetic analog of cerulenin $\mathrm{C} 75$ plus rosiglitazone where a $50 \%$ reduction in cell viability was obtained at $10.2 \mu \mathrm{M}$ final concentration. However, these drugs individually produced minimal effects on cell viability when used at 10.2 $\mu \mathrm{M}$ each (Fig. 5B). The additive anti-proliferative effects of rosiglitazone/FASN blocker combination was also evident in androgen independent PC-3 and DU-145 cells where cell viability was consistently lower in cells treated with rosiglitazone/C75 drug combination as compared to cells treated with rosiglitazone alone (Fig. 6). In principle, co-administration of TZDS and FASN blockers enhance the in vitro anti-tumor actions of each other.

Akin to our current findings, previous studies have shown additive benefits of combining PPAR $\gamma$ ligands with other anti-tumor agents. For instance, the tyrosine kinase inhibitor, gefitinib, enhanced the anti-proliferative effects of rosiglitazone when used for treatment of A549 lung cancer cells (9). The synergistic effect was attributed to rosiglitazone-induced genomic activation of PTEN, a tumor suppressor gene and a key negative regulator of the PI3K pathway that promotes growth in androgen-refractory PCa $(41,42)$. In the same cell line, the combinations of rosiglitazone with the platinumbased cytotoxic drugs carboplatin and cisplatin have synergistic inhibitory effects on A549 lung cancer cell growth (10). Gene array analysis has shown that rosiglitazone downregulates metallothioneins which are heavy metal binding proteins that mediate platinum drug resistance.

Our data indicates that the weaker anti-tumor activity of rosiglitazone, in contrast to stronger anti-tumor action of troglitazone, is likely due to rosiglitazone-induced genomic (via PPAR $\gamma$ activation) factors that negatively diminish the desirable non-genomic (apoptotic pathway) action. The 
enhanced PPAR $\gamma$-mediated expression of the anti-apoptotic enzyme FASN by low micromolar rosiglitazone concentration is a good example of genomic rosiglitazone-induced apoptosis modulating factor. This concept is supported by several observations in our data. First, in untreated cells FASN expression paralleled that of PPAR $\gamma$ in PC-3, DU-145 and LNCaP suggesting that overexpression of PPAR $\gamma$ in these cell lines could be contributing to parallel increased in the anti-apoptitic enzyme FASN. The strong correlation between PPAR $\gamma$ and FASN could result either from a direct upregulation of one gene by the other, or because the two genes could be similarly regulated by the existing physiological state of the cell. Second, the weaker anti-apoptotic effect of PPAR $\gamma$ agonist rosiglitazone in PC-3 cells (with highest PPAR $\gamma$ level) at lower concentration differ from the stronger effect of troglitazone suggesting involvement of PPAR $\gamma$-related factors in the weaker rosiglitazone effect. Third, rosiglitazone, a strong PPAR $\gamma$ ligand, induced over 7 -fold increase in FASN expression in LNCaP cells when used at low concentration. This finding apparently correlated with the lower susceptibility of FASN-overexpressing PC-3 cells to anti-apoptotic effect of rosiglitazone. Fourth, the FASN inhibitors cerulenin and its synthetic analog C75 abrogated the ability of rosiglitazone to induce FASN production and this finding could explain the enhancement of the antitumor activity obtained with rosiglitazone when combined with FASN inhibitors. Finally, other studies have convincingly shown that FASN overexpression protects normal human immortalized epithelial cells, and LNCaP PCa cells from apoptosis mediated via intrinsic pathways (25).

Our real-time PCR data showed that a low concentration of rosiglitazone $(5 \mu \mathrm{M})$ enhanced lipogenic gene production, whereas a higher micromolar concentration $(50 \mu \mathrm{M})$ caused activity repression of the same genes. This finding further supports the concept of a biphasic genomic (via PPAR $\gamma$ activation) and non-genomic (apoptotic) action of rosiglitazone in $\mathrm{PCa}$ where the apoptotic effect of rosiglitazone at high micromolar concentrations degrades the genomic response as evident by suppression or lack of changes in expression of all lipogenic genes. This biphasic effect provides a frame-work for dissociation of PPAR $\gamma$-dependent effects that stem from transcriptional activation of PPAR $\gamma$-responsive genes from PPAR $\gamma$-independent (non-genomic) effects. Further evidence in support of a rosiglitazone genomic and non-genomic actions include first, the lower susceptibility of PPAR $\gamma$ expressing PC-3 cells to anti-tumor effects of rosiglitazone at low rosiglitazone concentration when contrasted with the equal anti-tumor activity of rosiglitazone across all cell lines at higher micromolar concentrations. Second, the enhancement of rosiglitazone anti-tumor activity by PPAR $\gamma$ blocker at lower, but not higher micromolar concentrations, further suggests involvement of PPAR $\gamma$-mediated actions that contribute to the weaker rosiglitazone anti-tumor actions.

In general, the lack of anti-tumor effect of the PPAR $\gamma$ blocker GW 9662 is in agreement with previous findings (13) and suggests that the blockage of unstimulated PPAR $\gamma$ per se does not suppress or boost PCa cell growth. In contrast, the anti-tumor activity of rosiglitazone at low micromolar concentration is enhanced by pre-treatment with the PPAR $\gamma$ blocker presumably by preventing FASN production that could result from activation of PPAR $\gamma$ by rosiglitazone. The failure of GW 9662 to block the anti-tumor effect of troglitazone and rosiglitazone (at higher concentrations) adds further support to the concept of PPAR $\gamma$-independent anti-tumor effects of TZDs especially for troglitazone. The latter finding is also in agreement with the studies that have shown anti-tumor activity of TZDs analogs that lack PPAR $\gamma$ activity (2).

The enhanced anti-tumor effect of TZDs when combined with FASN inhibitors has important clinical significance since one of the major drawbacks of TZDs therapy in animals and human studies is the enhancement of lipogenesis and fat cell production (adipogenesis), leading to weight gain (33). The use of TZDs/FASN inhibitors could offer significant clinical advantage in blunting the undesirable weight gain (30) and growth promoting effects of FASN overexpression in cancer cells (40). Further, the TZDs/FASN combinations potently suppress LNCaP cell growth in the presence of DHT when compared with single drug treatment. This finding is significant because androgens play a pivotal role in growth and differentiation of the prostate epithelial and stromal cell compartments, and more importantly increase lipogenic gene expression including FASN production (38-40).

As evading apoptosis is one of the important hallmarks of cancer cell progression (43), data presented here shows that combining TZDs and FASN inhibitors lead to increased synergism in induction of apoptosis in PCa cells. This synergism may reduce the probability that tumor cells resistant to either type of agent will emerge. In summary, this approach should have clinical relevance in prostate and other cancers as newer members of TZDs and FASN inhibitors become available. Further studies are planned to investigate the in vivo anti-tumor effects of TZDs/FASN drug combinations in $\mathrm{PCa}$ animal models.

\section{Acknowledgements}

This study is partially supported by funds from the interdepartmental Research Grants Program, Scott-Richey Research Center and the Boshell Diabetes and Metabolic Diseases Program, College of Veterinary Medicine, Auburn University, Auburn, AL, USA.

\section{References}

1. Woolf SH: Screening for prostate cancer with prostate-specific antigen. An examination of the evidence. N Engl J Med 333: 1401-1405, 1995.

2. Shiau CW, Yang CC, Kulp SK, et al: Thiazolidenediones mediate apoptosis in prostate cancer cells in part through inhibition of Bcl-xL/Bcl-2 functions independently of PPARgamma. Cancer Res 65: 1561-1569, 2005.

3. Hisatake JI, Ikezoe T, Carey M, Holden S, Tomoyasu S and Koeffler HP: Down-regulation of prostate-specific antigen expression by ligands for peroxisome proliferator-activated receptor gamma in human prostate cancer. Cancer Res 60: 5494-5498, 2000

4. Yang CC, Wang YC, Wei S, et al: Peroxisome proliferatoractivated receptor gamma-independent suppression of androgen receptor expression by troglitazone mechanism and pharmacologic exploitation. Cancer Res 67: 3229-3238, 2007.

5. Wei S, Lin LF, Yang CC, et al: Thiazolidinediones modulate the expression of beta-catenin and other cell-cycle regulatory proteins by targeting the F-box proteins of Skp1-Cul1-F-box protein E3 ubiquitin ligase independently of peroxisome proliferator-activated receptor gamma. Mol Pharmacol 72: 725-733, 2007. 
6. Mueller E, Smith M, Sarraf P, et al: Effects of ligand activation of peroxisome proliferator-activated receptor gamma in human prostate cancer. Proc Natl Acad Sci USA 97: 10990-10995, 2000.

7. Hatton JL and Yee LD: Clinical use of PPARgamma ligands in cancer. PPAR Res 2008: 159415, 2008.

8. Karayi MK and Markham AF: Molecular biology of prostate cancer. Prostate Cancer Prostatic Dis 7: 6-20, 2004.

9. Lee SY, Hur GY, Jung KH, et al: PPAR-gamma agonist increase gefitinib's antitumor activity through PTEN expression. Lung Cancer 51: 297-301, 2006.

10. Girnun GD, Naseri E, Vafai SB, et al: Synergy between PPARgamma ligands and platinum-based drugs in cancer. Cancer Cell 11: 395-406, 2007.

11. Koeffler HP: Peroxisome proliferator-activated receptor gamma and cancers. Clin Cancer Res 9: 1-9, 2003.

12. Lee CH, Olson P and Evans RM: Minireview: Lipid metabolism, metabolic diseases, and peroxisome proliferator-activated receptors. Endocrinology 144: 2201-2207, 2003.

13. Chaffer CL, Thomas DM, Thompson EW and Williams ED: PPARgamma-independent induction of growth arrest and apoptosis in prostate and bladder carcinoma. BMC Cancer 6: $53,2006$.

14. Kuhajda FP, Pizer ES, Li JN, Mani NS, Frehywot GL and Townsend CA: Synthesis and antitumor activity of an inhibitor of fatty acid synthase. Proc Natl Acad Sci USA 97: 3450-3454, 2000.

15. Pflug BR, Pecher SM, Brink AW, Nelson JB and Foster BA: Increased fatty acid synthase expression and activity during progression of prostate cancer in the TRAMP model. Prostate 57: 245-254, 2003.

16. Menendez JA, Colomer R and Lupu R: Inhibition of tumorassociated fatty acid synthase activity enhances vinorelbine (Navelbine)-induced cytotoxicity and apoptotic cell death in human breast cancer cells. Oncol Rep 12: 411-422, 2004.

17. Debes JD and Tindall DJ: Mechanisms of androgen-refractory prostate cancer. N Engl J Med 351: 1488-1490, 2004.

18. Mashima T, Seimiya $H$ and Tsuruo T: De novo fatty-acid synthesis and related pathways as molecular targets for cancer therapy. Br J Cancer 100: 1369-1372, 2009.

19. Epstein JI, Carmichael M and Partin AW: OA-519 (fatty acid synthase) as an independent predictor of pathologic state in adenocarcinoma of the prostate. Urology 45: 81-86, 1995.

20. Kuhajda FP: Fatty acid synthase and cancer: new application of an old pathway. Cancer Res 66: 5977-5980, 2006.

21. Swinnen JV, Roskams T, Joniau S, et al: Overexpression of fatty acid synthase is an early and common event in the development of prostate cancer. Int J Cancer 98: 19-22, 2002.

22. Shurbaji MS, Kalbfleisch JH and Thurmond TS: Immunohistochemical detection of a fatty acid synthase (OA-519) as a predictor of progression of prostate cancer. Hum Pathol 27: 917-921, 1996.

23. Kuhajda FP: Fatty-acid synthase and human cancer: new perspectives on its role in tumor biology. Nutrition 16: 202-208, 2000.

24. Wakil SJ: Fatty acid synthase, a proficient multifunctional enzyme. Biochemistry 28: 4523-4530, 1989.

25. Migita T, Ruiz S, Fornari A, et al: Fatty acid synthase: A metabolicenzyme and candidate oncogene in prostate cancer. J Natl Cancer Inst 101: 519-532, 2009

26. De Schrijver E, Brusselmans K, Heyns W, Verhoeven G and Swinnen JV: RNA interference-mediated silencing of the fatty acid synthase gene attenuates growth and induces morphological changes and apoptosis of LNCaP prostate cancer cells. Cancer Res 63: 3799-3804, 2003.
27. Pizer ES, Chrest FJ, DiGiuseppe JA and Han WF: Pharmacological inhibitors of mammalian fatty acid synthase suppress DNA replication and induce apoptosis in tumor cell lines. Cancer Res 58: 4611-4615, 1998.

28. Pizer ES, Jackisch C, Wood FD, Pasternack GR, Davidson NE and Kuhajda FP: Inhibition of fatty acid synthesis induces programmed cell death in human breast cancer cells. Cancer Res 56: 2745-2747, 1996.

29. Liu B, Wang Y, Fillgrove KL and Anderson VE: Triclosan inhibits enoyl-reductase of type I fatty acid synthase in vitro and is cytotoxic to MCF-7 and SKBr-3 breast cancer cells. Cancer Chemother Pharmacol 49: 187-193, 2002.

30. Loftus TM, Jaworsky DE, Frehywot GL, et al: Reduced food intake and body weight in mice treated with fatty acid synthase inhibitors. Science 288: 2379-2381, 2000.

31. Mansour M, Coleman E, Dennis J, et al: Activation of PPARgamma by rosiglitazone does not negatively impact male sex steroid hormones in diabetic rats. PPAR Res 2009: 101857, 2009.

32. Vandesompele J, De Preter K, Pattyn F, et al: Accurate normalization of real-time quantitative RT-PCR data by geometric averaging of multiple internal control genes. Genome Biol 3: Research0034, 2002.

33. Watkins SM, Reifsnyder PR, Pan HJ, German JB and Leiter EH: Lipid metabolome-wide effects of the PPARgamma agonist rosiglitazone. J Lipid Res 43: 1809-1817, 2002.

34. Baron A, Migita T, Tang D and Loda M: Fatty acid synthase: a metabolic oncogene in prostate cancer? J Cell Biochem 91: 47-53, 2004.

35. Wei S, Yang J, Lee SL, Kulp SK and Chen CS: PPARgammaindependent antitumor effects of thiazolidinediones. Cancer Lett 276: 119-124, 2009.

36. Neuschwander-Tetri BA, Isley WL, Oki JC, et al: Troglitazoneinduced hepatic failure leading to liver transplantation. A case report. Ann Intern Med 129: 38-41, 1998.

37. Lupu R and Menendez JA: Targeting fatty acid synthase in breast and endometrial cancer: An alternative to selective estrogen receptor modulators? Endocrinology 147: 4056-4066, 2006.

38. Heemers H, Maes B, Foufelle F, Heyns W, Verhoeven G and Swinnen JV: Androgens stimulate lipogenic gene expression in prostate cancer cells by activation of the sterol regulatory elementbinding protein cleavage activating protein/sterol regulatory element-binding protein pathway. Mol Endocrinol 15: 1817-1828, 2001.

39. Dehm SM and Tindall DJ: Molecular regulation of androgen action in prostate cancer. J Cell Biochem 99: 333-344, 2006.

40. Swinnen JV, Heemers H, van de Sande T, et al: Androgens, lipogenesis and prostate cancer. J Steroid Biochem Mol Biol 92: 273-279, 2004.

41. Li J, Yen C, Liaw D, et al: PTEN, a putative protein tyrosine phosphatase gene mutated in human brain, breast, and prostate cancer. Science 275: 1943-1947, 1997.

42. Bertram J, Peacock JW, Tan C, et al: Inhibition of the phosphatidylinositol 3'-kinase pathway promotes autocrine Fas-induced death of phosphatase and tensin homologue-deficient prostate cancer cells. Cancer Res 66: 4781-4788, 2006.

43. Hanahan D and Weinberg RA: The hallmarks of cancer. Cell 100: 57-70, 2000. 